\title{
Wilms' tumor gene 1 enhances nutlin-3-induced apoptosis
}

\author{
SUN-YOUNG LEE ${ }^{1,3^{*}}$, YUN-JEONG CHOE ${ }^{1,3^{*}}$, JIK YOUNG PARK $^{4}$, SANG SUN LEE $^{1,3}$, \\ YOON-HYOUNG KIM ${ }^{1}$, SEOK JOON SHIN ${ }^{2}$, YEUN JUN CHUNG ${ }^{3}$ and HO-SHIK KIM ${ }^{1}$ \\ Departments of ${ }^{1}$ Biochemistry and ${ }^{2}$ Internal Medicine, ${ }^{3}$ Cancer Evolution Research Center, College of Medicine, \\ The Catholic University of Korea, Seoul 137-701; ${ }^{4}$ Seegene Institute of Life Science, \\ Seegene, Inc., Seoul 138-050, Republic of Korea
}

Received September 27, 2013; Accepted October 21, 2013

DOI: $10.3892 /$ or.2013.2832

\begin{abstract}
Nutlin-3, a human double minute 2 (HDM2) antagonist, induces cell cycle arrest or apoptosis by upregulating p53 in cancer cells. WT1, the product of Wilms' tumor gene 1 , has been shown to interact with p53, but the effect of WT1 on nutlin-3-induced apoptosis has yet to be examined. To address this issue, we analyzed the inhibitory effect of nutlin-3 on cell growth as a function of Wt1 expression status using a Wt1-inducible U2OS cell line. In the absence of Wt1 expression, nutlin-3 induced cell cycle arrest with marginal cytotoxicity. Furthermore, upon Wt1 expression, nutlin-3 exerted a marked degree of cell death, as evidenced by the accumulation of hypo-diploid cells and LDH release. During cell death induction, cytochrome $c$ was released into the cytosol, and caspase-9 and -3 were activated, suggesting that an intrinsic apoptotic pathway may be involved in this cell death. Consistent with this, z-VAD-Fmk, a pan-caspase inhibitor and the overexpression of $B C L-X L$ attenuated the cell death. Nutlin-3 caused an increase in the mRNA levels of both $B C L-X L$ and $B A K$, as well as their corresponding protein levels in mitochondria. In the presence of $\mathrm{Wt} 1$, nutlin-3-induced $B C L-X L$ expression was attenuated while the expression of nutlin-3-induced $B A K$ was potentiated. Collectively, these results suggest that WT1 potentiates nutlin-3-induced apoptosis by downregulating the expression of $B C L-X L$ while upregulating that of $B A K$, which leads to the activation of an intrinsic apoptotic pathway.
\end{abstract}

Correspondence to: Professor Ho-Shik Kim, Department of Biochemistry, College of Medicine, The Catholic University of Korea, 222 Banpo-daero, Socho-gu, Seoul 137-701, Republic of Korea

E-mail: hoshik@catholic.ac.kr; hoshik2013@gmail.com

${ }^{*}$ Contributed equally

Abbreviations: HDM2, human double minute 2; HSP70, heat shock protein 70; MTP, mitochondrial transmembrane potential; NUT3, nutlin-3; PARP1, poly(ADP-ribose) polymerase 1; Tet, tetracycline; WT1, Wilms' tumor gene 1

Key words: apoptosis, nutlin-3, tumor suppressor protein p53, Wilms' tumor gene 1 gene product

\section{Introduction}

Wilms' tumor gene WT1 is mutated in approximately $10-15 \%$ of sporadic Wilms' tumors (1). Germline mutations in WTI can lead to developmental diseases such as WAGR (Wilms tumor, aniridia, genitourinary abnormalities and intellectual disability), Denys-Drash and Frasier syndromes, all of which are characterized by glomerulonephropathy and abnormal gonad development (2). The importance of WT1 during development was further demonstrated using mouse models. Embryos of Wt1-/- mice die in utero with agenesis of the kidneys, gonads, adrenal glands and spleen $(3,4)$. WT1 encodes a Cys2-His2 (C2H2) zinc finger protein that functions as a transcription factor (5). WT1 has two alternative splicing sites, three translational initiation sites, and one RNA editing site, making possible the generation of at least 24 isoforms in the cell. The first alternative splicing introduces 17 amino acids encoded by exon 5 and the second alternative splicing inserts or omits three amino acids, KTS (Lys, Thr and Ser) between the third and fourth zinc fingers. Since KTS insertion by second alternative splicing disrupts the spacing between two zinc fingers, the +KTS isoform of WT1 has an altered DNA binding specificity.

Developmental processes are closely related to cell death. During the embryonic development of the kidney, metanephric mesenchyme serves as a precursor of glomerular and tubular structures. Wt1-null mice develop extensive apoptosis in the metanephric blastema (3) suggesting that WT1 protects renal precursor cells from apoptosis. Bcl-2 expression by WT1 can explain its anti-apoptotic function during kidney development (6). Consistent with the anti-apoptotic effects during development, WT1 promotes the survival of chronic leukemic cells such as K562 and MM6 (7). However, the expression of +KTS isoforms (+Ex5/+KTS and -Ex5/+KTS) of WT1 induces apoptosis in HepG2 cells (8). Another isoform of WT1, -Ex5/-KTS, induces apoptosis in Saos-2 cells by upregulating $B A K$ expression (9). These findings suggest that WT1 may have a dichotomous effect on cancer cell death depending on the cell types and the isoforms. One of the characteristic features of WT1 is its interactions with several proteins that function in apoptosis, such as p53 and HSP70 $(10,11)$. Maheswaran et al showed that WT1 stabilizes p53 (10) and inhibits UV-induced apoptosis which is dependent on $\mathrm{p} 53$, but not p53-induced cell cycle arrest (12). The molecular mecha- 
nisms that are involved in the effect of WT1 on p53-induced apoptosis, however, remain to be elucidated.

Nutlin-3 is a small molecule that binds to the p53 binding pocket of HDM2 which ubiquitylates and degrades p53 (13). Thus, nutlin-3 elevates the level of expression of p53 and activates the $\mathrm{p} 53$ pathway. In cancer cells that harbor wildtype p53, nutlin-3 can activate p53-dependent growth arrest or an apoptosis program (14). Based on the ability of WT1 to interact and modulate the activity of p53, we investigated whether WT1 also modulates the anticancer effect of nutlin-3. To address this issue, we analyzed the effect of nutlin-3 on the growth and survival of U2OS cells which express $W t 1$ according to the presence of tetracycline.

\section{Materials and methods}

Chemicals. Nutlin-3 was purchased from Sigma-Aldrich (St.Louis, MO,USA).Z-VAD-Fmk was from Tocris Bioscience (Northpoint, UK). Unless otherwise specified, all chemicals were of molecular biology grade and were purchased from Sigma-Aldrich.

Cell culture. The UD29a cell line was stably established from a U2OS cell line (human osteosarcoma cells; ATCC, Manassas, VA, USA) by transfection of $W t 1(+E x 5 /+K T S)$ regulated by tetracycline-repressible promoter and maintained in DMEM (Invitrogen, Carlsbad, CA, USA) supplemented with 10\% heatinactivated fetal bovine serum (HyClone, Logan, UT, USA), $100 \mathrm{U} / \mathrm{ml}$ of penicillin/streptomycin (Invitrogen) and $1 \mu \mathrm{g} / \mathrm{ml}$ of tetracycline under a $5 \% \mathrm{CO}_{2}$ and $95 \%$ humidified atmosphere. To induce the expression of $W t 1$, cells were washed three times with calcium, magnesium-free phosphate-buffered saline (Invitrogen) and replenished with DMEM without tetracycline.

Cell death assessment. Cell viability was measured by the MTT [3-(4,5-dimethylthiazol-2-yl)-2,5-diphenyltetrazolium bromide] reduction assay. UD29a cells were seeded at a density of $5 \times 10^{4}$ cells/well in 24-well plates and after $24 \mathrm{~h}$, tetracycline was removed and nutlin-3 was added. Following incubation for the indicated periods, MTT was added and the optical density of each well was measured at $570 \mathrm{~nm}$. Data are expressed as relative cell survival which represents the percentage of the optical density of cells treated with nutlin-3 to that of non-treated cells. For the measurement of apoptotic cells, UD29a cells treated with nutlin-3 were fixed in 70\% ethanol and stained with propidium iodide (PI) followed by flow cytometric analysis (FACSCalibur, BD Biosciences, San Jose, CA, USA) as previously reported (15). Cell cycle distribution was analyzed by CellQuest and ModFit software.

Immunoblot analysis. Cell lysates, mitochondrial fractions or cytosolic fractions were subjected to SDS-PAGE and immunoblot analysis following a previous method (16). The proteins were then transferred onto a nitrocellulose transfer membrane (Whatman, Dassel, Germany). After the transfer, membranes were blocked for $30 \mathrm{~min}$ in TTBS buffer ( $25 \mathrm{mM}$ Tris, $\mathrm{pH} 7.4$, $138 \mathrm{mM} \mathrm{NaCl}, 0.05 \%$ Tween-20) containing 5\% skim milk and then incubated with indicated antibodies. After washing four times with TTBS, the membranes were incubated with antimouse IgG-peroxidase conjugated. The membranes were then washed four times in TTBS, followed by chemiluminescencebased detection (GE Healthcare, Buckinghamshire, UK).

Subcellular fractionation. Cytosolic and mitochondrial fractions were separated as described in a previous report (17). Briefly, cells were treated as indicated and were resuspended in ice-cold hypotonic buffer (10 $\mathrm{mM}$ Hepes, $10 \mathrm{mM} \mathrm{MgCl}{ }_{2}$, $42 \mathrm{mM} \mathrm{KCl}$ ) containing a protease inhibitor cocktail. Plasma membranes were ruptured by passing the cells through a 31 -gauge syringe. Following centrifugation at 1,000 rpm for $5 \mathrm{~min}$ at $4^{\circ} \mathrm{C}$, the supernatant was collected and again centrifuged at $13,000 \mathrm{rpm}$ for $15 \mathrm{~min}$ at $4^{\circ} \mathrm{C}$. The clear fraction was saved as the cytosolic fraction and the pellet, the heavy membrane fraction, was used as the mitochondrial fraction.

Assay of mitochondrial transmembrane potential (MTP). The change in MTP during apoptosis was analyzed using a MitoProbe $^{\mathrm{TM}}$ JC-1 Assay kit (Invitrogen), following the manufacturer's instructions.

Real-time quantitative RT-PCR ( $q R T-P C R)$. Total RNA that was extracted from cells treated as indicated was reverse transcribed to cDNA using a random hexamer and CycleScript Reverse Transcriptase (Bioneer Corp., Daejeon, Korea) and subjected to qRT-PCR. qRT-PCR was performed with a mixture containing cDNA, SYBR Premix Ex Taq (Takara Bio Inc., Otsu, Japan) and primers using Mx3000P QPCR System (Stratagene, La Jolla, CA, USA). The level of change in gene expression change was calculated by the $\Delta \Delta \mathrm{Ct}$ method using GAPDH as an internal control (18). All tests were carried out in triplicate and repeated three times.

Expression of human BCL-XL. Coding sequence of human BCL-XL was amplified using ExTaq DNA polymerase (Takara Bio) and inserted into pcDNA3.1-vector (Invitrogen). After the nucleotide sequence of BCL-XL in pcDNA3.1-/BCL-XL was confirmed to be completely matched with published data (GenBank accession no. NM_138578), pcDNA3.1-empty vector or pcDNA3.1-/BCL-XL was transfected into UD29a cells using FuGENE 6 transfection reagent.

\section{Results}

Potentiation of nutlin-3-induced growth suppression by Wt expression. Among all the isoforms of WT1, +Ex5/+KTS is the most abundant, accounting for $>60 \%$ of all WT1 transcripts (19). Thus, we first examined the effect of this isoform on the nutlin-3-mediated activation of $\mathrm{p} 53$. As shown in Fig. 1A, the addition of nutlin-3 led to a marked increase in the level of $\mathrm{p} 53$ protein and the expression of $\mathrm{Wt} 1$ did not further increase $\mathrm{p} 53$ protein levels induced by nutlin-3. However, the presence of nutlin-3 also caused an increase in Wt1 levels (compare lanes 3 and 4). Consistent with its function as an HDM2 inhibitor, nutlin-3 had no effect on p53 mRNA levels, while the level of p21WAF1 mRNA increased in parallel with that for the p53 protein, indicating that p53 is functionally intact in this cell line (Fig. 1B). Nutlin-3 treatment inhibited the growth of UD29a cells, accompanied by a marginal release of lactate dehydrogenase (LDH) (Fig. 1C). The expression of Wt1 by itself had no negative effects on cell growth but, 
A

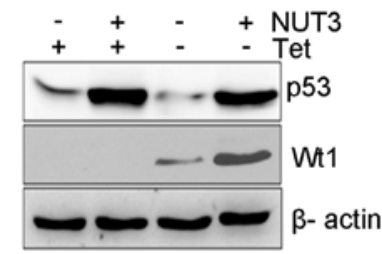

B

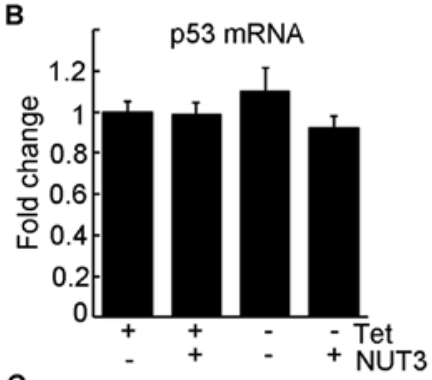

C


Figure 1. Effect of Wt1 on nutlin-3-induced growth inhibition. UD29a cells were treated with $20 \mu \mathrm{M}$ nutlin-3 (NUT3) in the absence or presence of Wt1 (+Ex5/+KTS) for $48 \mathrm{~h}$. (A) Lysates of UD29a cells were subjected to immunoblot analysis. $\beta$-actin was used for equal loading of proteins. (B) Total RNAs of UD29a cells were extracted and subjected to qRT-PCR against $p 53$ and $p 21 W A F 1$ as described in Materials and methods. All data represent the means \pm SD of three independent experiments, each sample of which was measured in triplicate. (C, left panel) Cell viability was determined by MTT assay and expressed as relative cell survival which indicates the percentage of cell viability of the NUT3-treated cells to that of the cells grown in the presence of $1 \mu \mathrm{g} / \mathrm{ml}$ tetracycline (Tet) without $20 \mu \mathrm{M}$ NUT3. (C, right panel) Culture media of UD29a cells were collected and subjected to LDH release assay according to the manufacturer's instruction. LDH release of each group was normalized to that of the non-treated group and expressed as foldchange. All data represent the means \pm SD of three independent experiments performed in triplicate.

in the presence of nutlin-3, growth suppression was further potentiated and the release of LDH was markedly increased (Fig. 1C), suggesting that the combination of WT1 expression and nutlin-3 treatment leads to enhanced cell death.

Activation of caspase-dependent apoptosis by nutlin-3 in the presence of $W t 1$. To examine the mechanism responsible for cell death induction, we analyzed the cell cycle distribution and the activation of caspases. As shown in Fig. 2A, nutlin-3 treatment alone induced a decrease in S-phase cells, suggesting p53-mediated delay or arrest of cell cycle progression. In the presence of WT1, nutlin-3 induced a striking increase in the sub-G1 population of UD29a cells but not non-WT1-expressing cells. Parallel to the level of the sub-G1 population, nutlin-3 induced the activation of caspase- 9 and -3 , and the cleavage of poly(ADP-ribose) polymerase 1 (PARP1) (Fig. 2B). In addition, this cell death was diminished by pretreatment with z-VAD-Fmk, a pan-caspase inhibitor
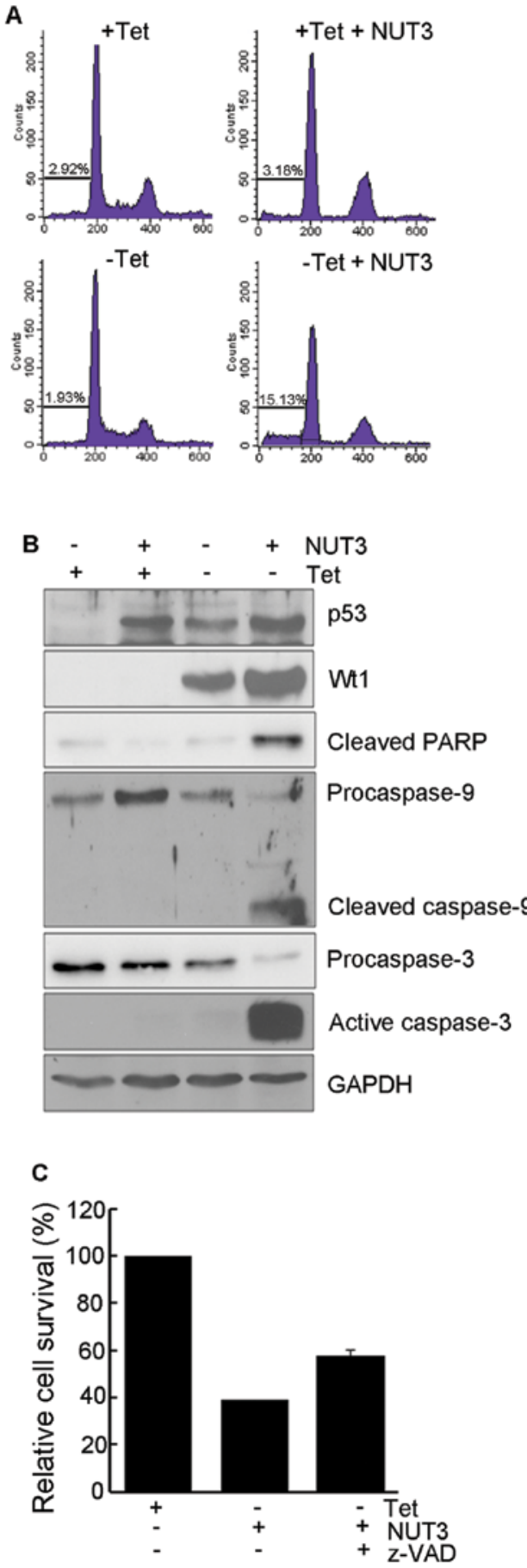

Figure 2. The effect of Wt1 on nutlin-3-induced apoptosis. (A) UD29a cells treated with $20 \mu \mathrm{M}$ nutlin-3 (NUT3) in the presence or absence of $1 \mu \mathrm{g} / \mathrm{ml}$ tetracycline (Tet) for $48 \mathrm{~h}$ were fixed, stained with propidium iodide (PI) and analyzed by flow cytometry. (B) Lysates of UD29a cells treated as indicated for $48 \mathrm{~h}$ were subjected to immunoblot analysis against indicated proteins. GAPDH was used for equal loading of proteins. (C) UD29a cells incubated with or without $50 \mu \mathrm{M}$ z-VAD-Fmk (z-VAD) for $1 \mathrm{~h}$ were treated with NUT3 in the absence of Tet. At $24 \mathrm{~h}$ post-treatment of nutlin-3, MTT assay was performed and expressed as relative cell survival (\%) which indicates the percentage of cell viability of the NUT3-treated cells to that of the cells grown in the presence of $1 \mu \mathrm{g} / \mathrm{ml}$ Tet without $20 \mu \mathrm{M}$ NUT3. Data represent the means \pm SD of three independent experiments performed in triplicate.

(Fig. 2C). Collectively, these results suggest that nutlin-3 induces apoptosis via an intrinsic apoptosis pathway in the presence of WT1. 
A



B


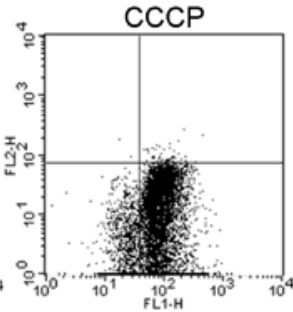

Figure 3. Activation of the intrinsic apoptotic pathway by nutlin-3 and Wt1. (A) Cytosolic and mitochondrial fractions of UD29a cells treated as indicated for $48 \mathrm{~h}$ were subjected to immunoblot analysis against cytochrome $c$ (Cyt C). GAPDH and cytochrome $c$ oxidase (Cyt C Oxidase) were used for equal loading of cytosolic and mitochondrial fractions, respectively. (B) UD29a cells treated as indicated for $48 \mathrm{~h}$ or $50 \mu \mathrm{M}$ CCCP for 5 min were incubated with $2 \mu \mathrm{M}$ JC-1 using a MitoProbe ${ }^{\mathrm{TM}}$ JC-1 Assay kit. After 15 min, cells were harvested and analyzed on FACSCalibur. CCCP was used to confirm that JC-1 sensitively responded to MTP dissipation. These data are representative of three independent experiments.
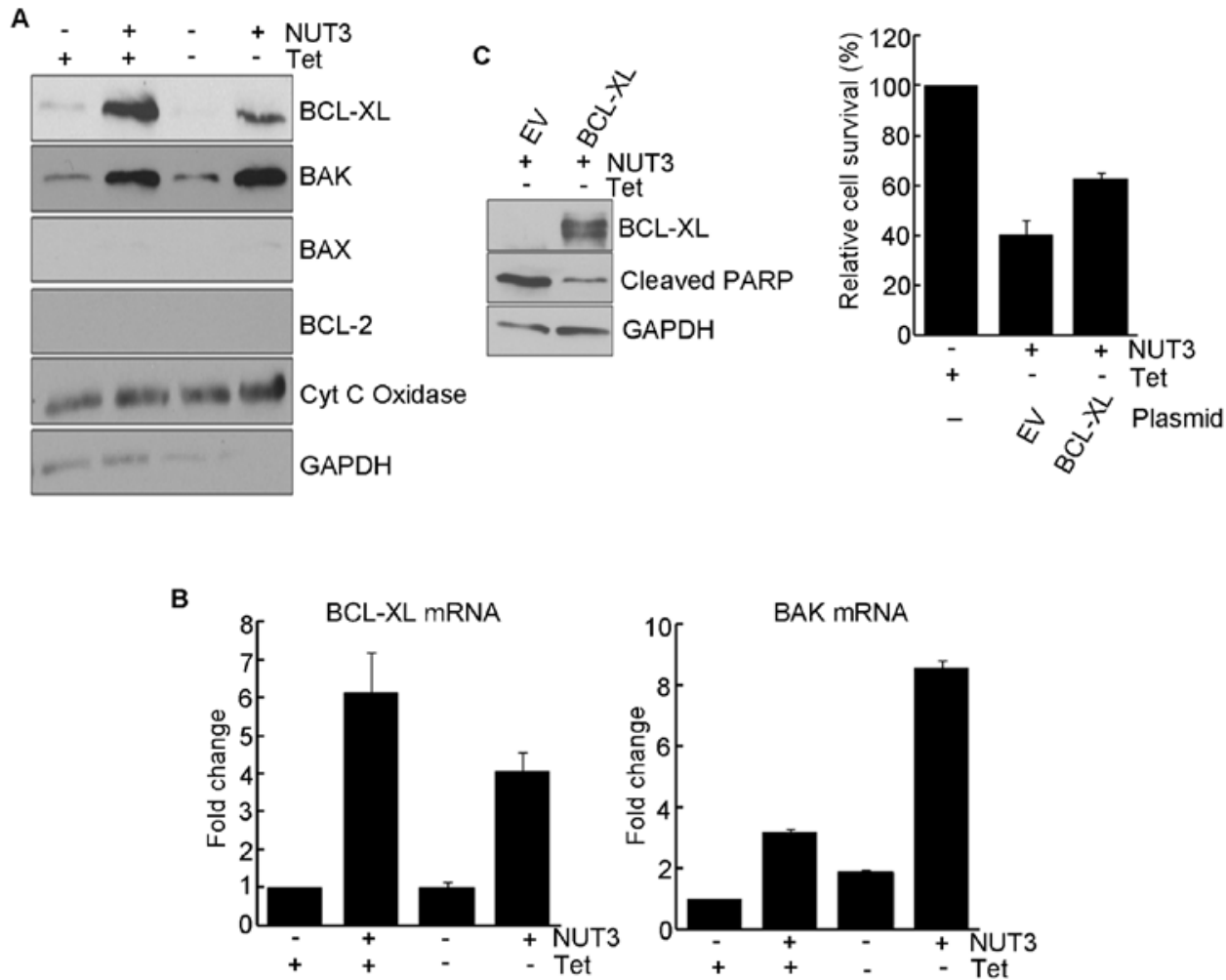

Figure 4. The effect of Wt1 on the nutlin-3-induced accumulation of BAK and BCL-XL. (A and B) UD29a cells were treated with $20 \mu \mathrm{M}$ nutlin-3 (NUT3) in the presence or absence of $1 \mu \mathrm{g} / \mathrm{ml}$ tetracycline (Tet) for $48 \mathrm{~h}$. (A) Mitochondrial fractions of UD29a cells were subjected to immunoblot analysis. Cytochrome $c$ oxidase (Cyt C Oxidase) was used for equal loading of mitochondrial proteins. (B) Total RNAs of cells treated as indicated were extracted and subjected to qRT-PCR of $B C L-X L$ and $B A K$ mRNAs using $G A P D H$ as the internal control. The data shown are the means $\pm \mathrm{SD}$ of three independent experiments performed in triplicate. (C) UD29a cells transfected with pCDNA3.1(23) (EV) or pCDNA3.1(23)/BCL-XL (BCL-XL) for $24 \mathrm{~h}$ were treated with $20 \mu \mathrm{M}$ NUT3 in the absence of Tet. At $48 \mathrm{~h}$ post-treatment, cell lysates were subjected to immunoblot analysis (left panel), and MTT assay was performed and relative cell survival was calculated (right panel). The data represent the means $\pm \mathrm{SD}$ of three independent experiments performed in triplicate.

Release of cytochrome c by nutlin-3 and Wt1 expression. Since the intrinsic apoptotic pathway is activated by cytochrome $c$ released into the cytosol, we measured the intracellular localization of cytochrome $c$. The addition of nutlin-3 alone did not lead to the release of cytochrome $c$ into the cytosol (Fig. 3A), which is consistent with the absence of any activation of caspases and PARP1 cleavage (Fig. 2B). When nutlin-3 was added in the presence of WT1 expression, however, a marked 
increase in the cytosolic cytochrome $c$ was observed. The release of cytochrome $c$ was not associated with the alteration of the MTP (Fig. 3B), indicating that the cytochrome $c$ is released through pores or channels formed in the mitochondrial outer membranes.

The expression of $B C L-X L$ and $B A K$ during cell death. It is well known that MTP-independent cytochrome $c$ release occurs through the pores formed by BAX or BAK (20). Therefore, we examined the expression of BCL-2 family proteins in mitochondria after nutlin-3 treatment. As shown in Fig. 4A, nutlin-3 treatment resulted in an increase in the expression of BCL-XL and BAK while the expression of BCL-2 and BAX was not observed. In UD29a cells expressing Wt1 and treated with nutlin-3, a sharp decrease in BCL-XL and a modest increase in BAK occurred at the protein level. At the mRNA level, nutlin-3 treatment led to significant increases in $B C L-X L$ (6-fold) and $B A K$ (3-fold) transcripts. The expression of $W t 1$ alone led to a modest induction (<2-fold) of $B A K$ mRNA but had no effect on the expression of $B C L-X L$ (Fig. 4B). The expression of $W t 1$ in UD29a cells treated with nutlin-3 resulted in a synergistic increase (8-fold) in the expression of $B A K$ transcripts while Wt1 attenuated the nutlin-3-induced expression of $B C L-X L$ transcripts. The overexpression of $B C L-X L$ in Wt1-expressing cells attenuated nutlin-3-induced apoptosis, as evidenced by a significant increase in cell survival and a decreased level of cleaved PARP1. These results suggest that WT1 potentiates nutlin-3-induced apoptosis by the synergistic induction of $B A K$ expression and a simultaneous decrease in $B C L-X L$ expression.

\section{Discussion}

p53 is a critical activator of apoptosis which is induced by DNA damaging agents such as $\gamma$-irradiation and UV. In contrast to the report that WT1 inhibits UV-induced apoptosis, the + Ex $5 /+$ KTS isoform of Wt1 potentiated nutlin-3-induced apoptosis in the present study. In the absence of Wt1 expression, cell cycle arrest with marginal cytotoxicity was induced by nutlin-3, but in the presence of Wt1 (+Ex5/+KTS) expression, nutlin-3 treatment provoked overt cell death (Fig. 1C). Based on an analysis of cell cycle distribution (Fig. 2A) and biochemical markers (Fig. 2B), cell death induced by nutlin-3 in Wt1-expressing cells appears to be mediated by apoptosis. During the induction of apoptosis in Wt1-expressing cells, cytochrome $c$ was released into the cytosol in an MTP-independent manner (Fig. 3) and both caspase-9 and -3 were activated (Fig. 2B), demonstrating that the intrinsic apoptotic pathway played a major role in this model.

BCL-2 family proteins are essential players in MTP-independent release of cytochrome $c$. Both BCL-XL and BAK were found to have accumulated in mitochondria of nutlin3-treated cells (Fig. 4A). Notably, when Wt1 was expressed, nutlin-3-induced $B C L-X L$ and $B A K$ expression was reduced and augmented, respectively (Fig. 4A), which would likely result in the activation of BAK. BAK can make pores or channels in mitochondrial outer membranes through which cytochrome $c$ can exit into the cytosol, initiating the formation of apoptosome (19). The release of cytochrome $c$ by a combination of nutlin-3 treatment and Wt1 $(+E \times 5 /+K T S)$ expression was reversed by the overexpression of $B C L-X L$ (Fig. 4C), which further supports the involvement of BAK in this process. The increase in BCL-XL and BAK by nutlin-3 was accompanied with that of transcripts (Fig. 4B), indicating p53-mediated transcriptional activation. Although they are not generally considered to be transcriptional target genes of p53, and there has been no report showing that p53 stimulates the transcription of $B C L-X L$, it was reported that the transcription of $B A K$ can be induced by $\mathrm{p} 53$, although to a lesser extent than that for $p 21 W A F 1$ and $B A X(21)$. Therefore, it is possible that $B C L-X L$ and $B A K$ genes contain p53-responsive elements, albeit very weak, in their promoters, and a gradual accumulation of p53 by nutlin-3 would eventually lead to a recruitment of p53 to these promoters, finally resulting in a transcriptional increase in this cell line.

It is noteworthy that Wt1 displayed opposing effects on nutlin-3-induced $B C L-X L$ and $B A K$ expression (Fig. 4A and $B$ ). Consistent with the report that the -Ex5/-KTS isoform of Wt1 stimulated the transcription of $B A K$ in Saos-2 cells through direct binding to a $B A K$ promoter (9), we observed a modest (<2-fold) induction in $B A K$ transcription by the $W t 1$ + Ex5/+KTS isoform (Fig. 4B). Moreover, the finding that the level of Wt1 protein was upregulated by nutlin-3 (Fig. 1A) may suggest that the induction of $B A K$ transcription by Wt1 may be surged via upregulated Wt1 protein and thus increase of its transcriptional activity. However, since WT1 +KTS has a lower DNA binding affinity compared to the -KTS isoform, it is also reasonable to expect that WT1 +KTS may modulate the p53-induced expression of $B C L-X L$ and $B A K$ expression though its interaction with p53 or by regulating signal transduction pathways that are activated by nutlin-3. Considering that the activity of p53 has been shown to be regulated by several coregulators and that WT1 was also reported to act as a coregulator of p53 (10), it can be assumed that WT1 binds to p53 and affects p53-induced $B A K$ and $B C L-X L$ transcription by altering the interaction of cofactors with p53. p53 can also activate apoptosis by a non-transcriptional mechanism. It has been reported that p53 can be translocated to mitochondrial membranes and interact with BAK (22), BAX (23) or BCL-XL (24), thus enhancing the channel-forming activities of BAK and BAX. WT1, which was originally characterized as a nuclear protein, has been shown to shuttle between the cytosol and the nucleus $(25,26)$. These reports might also indicate that, in the transcription-independent activation of apoptosis, WT1 binds to p53 or protein complexes containing p53 in the nucleus as well as the cytosol, and moves to mitochondrial membranes to potentiate the p53-mediated apoptotic activity. Therefore, to identify the mechanism responsible for how WT1 potentiates nutlin-3-induced apoptosis, the mechanisms involved in $B A K$ and $B C L-X L$ induction as well as BAK activation in mitochondria by a combination of nutlin-3 treatment and WT1 expression need to be studied further.

Although nutlin-3 can induce cell cycle arrest and apoptosis, it has been reported to predominantly induce mitotic arrest in solid tumor cell lines (27). Mitotic arrest can contribute to the inhibition of cancer growth, but from the view point of chemotherapeutic efficiency, it diminishes apoptosis and promotes an acquired resistance to anticancer therapeutics (28). Therefore, it can be expected that molecules such as the WT1 protein that enhance the apoptosis by nutlin-3 
can be applied to cancer treatment as combined with nutlin-3. For the development of WT1 as an enhancer of the anticancer effect of nutlin-3, the mechanism underlying the potentiation of p53-induced apoptosis by WT1 and whether the effect of WT1 is universal or cell type- or isoform-specific should be determined first. Eventually, the molecular characterization of relationships between WT1 and p53 could contribute to the establishment of a carcinogenesis model and the development of a new treatment modality against cancer in the future.

\section{Acknowledgements}

The authors thank Dr Sean B. Lee for his critical comments and suggestions. This research was supported by a grant (2012R1A5A2047939) and a Basic Science Research Program through the National Research Foundation of Korea (NRF2010-0025420).

\section{References}

1. Hastie ND: Wilms' tumour gene and function. Curr Opin Genet Dev 3: 408-413, 1993.

2. Call KM, Glaser T, Ito CY, et al: Isolation and characterization of a zinc finger polypeptide gene at the human chromosome 11 Wilms' tumor locus. Cell 60: 509-520, 1990.

3. Kreidberg JA, Sariola H, Loring JM, et al: WT-1 is required for early kidney development. Cell 74: 679-691, 1993.

4. Herzer U, Crocoll A, Barton D, Howells N and Englert C: The Wilms tumor suppressor gene wt1 is required for development of the spleen. Curr Biol 9: 837-840, 1999.

5. Lee SB and Haber DA: Wilms tumor and the WT1 gene. Exp Cell Res 264: 74-99, 2001.

6. Mayo MW, Wang CY, Drouin SS, et al: WT1 modulates apoptosis by transcriptionally upregulating the bcl-2 proto-oncogene. EMBO J 18: 3990-4003, 1999.

7. Algar EM, Khromykh T, Smith SI, Blackburn DM, Bryson GJ and Smith PJ: A WT1 antisense oligonucleotide inhibits proliferation and induces apoptosis in myeloid leukaemia cell lines. Oncogene 12: 1005-1014, 1996.

8. Menke AL, Shvarts A, Riteco N, van Ham RC, van der Eb AJ and Jochemsen AG: Wilms' tumor 1-KTS isoforms induce p53-independent apoptosis that can be partially rescued by expression of the epidermal growth factor receptor or the insulin receptor. Cancer Res 57: 1353-1363, 1997.

9. Morrison DJ, English MA and Licht JD: WT1 induces apoptosis through transcriptional regulation of the proapoptotic $\mathrm{Bcl}-2$ family member Bak. Cancer Res 65: 8174-8182, 2005.

10. Maheswaran S, Park S, Bernard A, et al: Physical and functional interaction between WT1 and p53 proteins. Proc Natl Acad Sci USA 90: 5100-5104, 1993.
11. Maheswaran S, Englert C, Zheng G, et al: Inhibition of cellular proliferation by the Wilms tumor suppressor WT1 requires association with the inducible chaperone Hsp70. Genes Dev 12: 1108-1120, 1998

12. Maheswaran S, Englert C, Bennett P, Heinrich G and Haber DA: The WT1 gene product stabilizes p53 and inhibits p53-mediated apoptosis. Genes Dev 9: 2143-2156, 1995.

13. Vassilev LT, Vu BT, Graves B, et al: In vivo activation of the p53 pathway by small-molecule antagonists of MDM2. Science 303: 844-848, 2004.

14. Shangary S and Wang S: Small-molecule inhibitors of the MDM2-p53 protein-protein interaction to reactivate $\mathrm{p} 53$ function: a novel approach for cancer therapy. Annu Rev Pharmacol Toxicol 49: 223-241, 2009.

15. Lee K, Lee MH, Kang YW, Rhee KJ, Kim TU and Kim YS: Parkin induces apoptotic cell death in TNF- $\alpha$-treated cervical cancer cells. BMB Rep 45: 526-531, 2012.

16. Wang Z, Zhang Z, Wu Y, et al: Effects of echinomycin on endothelin-2 expression and ovulation in immature rats primed with gonadotropins. Exp Mol Med 44: 615-621, 2012.

17. Lee SY, Shin SJ and Kim HS: ERK1/2 activation mediated by the nutlin-3-induced mitochondrial translocation of p53. Int J Oncol 42: 1027-1035, 2013.

18. Livak KJ and Schmittgen TD: Analysis of relative gene expression data using real-time quantitative PCR and the $2^{-\Delta \Delta \mathrm{Ct}}$ method. Methods 25: 402-408, 2001.

19. Haber DA, Sohn RL, Buckler AJ, Pelletier J, Call KM and Housman DE: Alternative splicing and genomic structure of the Wilms tumor gene WT1. Proc Natl Acad Sci USA 88: 9618-9622, 1991.

20. Green DR and Kroemer G: The pathophysiology of mitochondrial cell death. Science 305: 626-629, 2004.

21. Zhao R, Gish K, Murphy M, et al: Analysis of p53-regulated gene expression patterns using oligonucleotide arrays. Genes Dev 14: 981-993, 2000.

22. Leu JI, Dumont P, Hafey M, Murphy ME and George DL: Mitochondrial p53 activates Bak and causes disruption of a Bak-Mcl1 complex. Nat Cell Biol 6: 443-450, 2004.

23. Chipuk JE, Kuwana T, Bouchier-Hayes L, et al: Direct activation of Bax by p53 mediates mitochondrial membrane permeabilization and apoptosis. Science 303: 1010-1014, 2004.

24. Mihara M, Erster S, Zaika A, et al: p53 has a direct apoptogenic role at the mitochondria. Mol Cell 11: 577-590, 2003.

25. Vajjhala PR, Macmillan E, Gonda T and Little M: The Wilms tumour suppressor protein, WT1, undergoes CRM1-independent nucleocytoplasmic shuttling. FEBS Lett 554: 143-148, 2003.

26. Niksic M, Slight J, Sanford JR, Caceres JF and Hastie ND: The Wilms' tumour protein (WT1) shuttles between nucleus and cytoplasm and is present in functional polysomes. Hum Mol Genet 13: 463-471, 2004.

27. Tovar C, Rosinski J, Filipovic Z, et al: Small-molecule MDM2 antagonists reveal aberrant p53 signaling in cancer: implications for therapy. Proc Natl Acad Sci USA 103: 1888-1893, 2006.

28. Moreno CS, Matyunina L, Dickerson EB, et al: Evidence that p53-mediated cell-cycle-arrest inhibits chemotherapeutic treatment of ovarian carcinomas. PLoS One 2: e441, 2007. 